\title{
Effects of chronic airway disease on health status of geriatric patients
}

\author{
Raffaele Antonelli Incalzi ${ }^{1}$, Riccardo Pistelli ${ }^{1}$, Claudio Imperiale ${ }^{2}$, Filippo Catalano ${ }^{3}$, Nicola \\ Scichilone ${ }^{3}$, Vincenzo Bellia ${ }^{3}$, and the Sa.R.A. Study Group* \\ ${ }^{1}$ Istituto di Medicina Interna e Geriatria, Catholic University, Roma, ${ }^{2}$ Fondazione San Raffaele - Cittadella \\ della Carità, Taranto, ${ }^{3}$ Istituto di Medicina Generale e Pneumologia, University of Palermo, Palermo, Italy
}

ABSTRACT. Background and aims: The impact of chronic airway disease on the health status of elderly patients is only to some extent explained by indexes of airflow limitation. The present study was designed to assess to what extent: 1) asthma, chronic obstructive pulmonary disease (COPD) and chronic bronchitis with normal FEV1 (simple bronchitis) differ in their impact on health status; 2) health status depends upon non-respiratory factors. Methods: A total of 1601 outpatients over 65 - 198 with asthma, 228 with COPD, 91 with simple bronchitis, and 1084 with non-respiratory illnesses (control group) were studied by collection of five health status indexes and multidimensional assessment. Discriminant analysis was used to identify health status profiles of groups. Demographic, anthropometric, clinical and respiratory function correlates of selected health status profiles were identified. Results: Only 26 and $28 \%$ of asthma and COPD patients vs $43 \%$ of simple bronchitis and $50 \%$ of non-respiratory patients showed group-specific health status profiles. These profiles were characterized by lower 6 min walked distance and greater index of disturbed sleep in asthmatics, and by worse performance on Barthel Index, 6-min walking test and Mini-Mental State Examination in COPD patients. More severe bronchial obstruction, a greater index of comorbidity and a longer occiput-wall distance characterized COPD patients with the worst health status. Conclusions: The health status of elderly patients with COPD or asthma is highly heterogeneous. On average, COPD is characterized by more severe physical impairment, and asthma by poorer quality of sleep. Comorbidity and severity of bronchial obstruction, but not age, contribute toward defining a subset of COPD patients with the worst health status.

(Aging Clin Exp Res 2004; 16: 26-33)

${ }^{\circ}$ 2004, Editrice Kurtis

\section{INTRODUCTION}

Health status encompasses health-related quality of life and functional status (1). Instruments assessing health status are commonly defined as health outcomes because they are primarily devoted to measuring the outcome of health care. Moreover, health outcomes can improve the prediction of clinical outcomes, such as mortality and onset of disability, as well as the use of resources in various clinical settings (2-7).

Both generic and disease-specific instruments assessing health outcomes have been shown to be weak correlates of Forced Expiratory Volume in the first second (FEV1) and Forced Vital Capacity (FVC) in patients with chronic airflow obstruction (8). Furthermore, pharmacological or rehabilitation therapy can improve health outcomes, as assessed by an instrument assessing health-related quality of life, even in the absence of any measurable effect on respiratory function indexes $(9,10)$. The highly subjective dimension of health-related quality of life and its dependence on the coping behavior and comorbidity in elderly patients with chronic obstructive pulmonary disease (COPD) may explain how perceived health status improves in the absence of measurable changes in respiratory function and physical performance (11-13). Analogously, dyspnoea and depression, but not forced expiratory volume, contribute to explaining most of the variance in a disease-specific health status index in a population of elderly asthmatics (14).

The present study was designed to assess health sta-

\footnotetext{
*See Appendix for the list of contributors.

Key words: Asthma, comorbidity, COPD, elderly, health status.

Correspondence: R. Antonelli Incalzi, MD, Istituto di Medicina Interna e Geriatria, Università Cattolica del Sacro Cuore, Largo A. Gemelli 8, 00168 Roma, Italy.

E-mail: raffaele_antonelli@rm.unicatt.it

Received January 13, 2003; accepted in revised form September 11, 2003.
} 


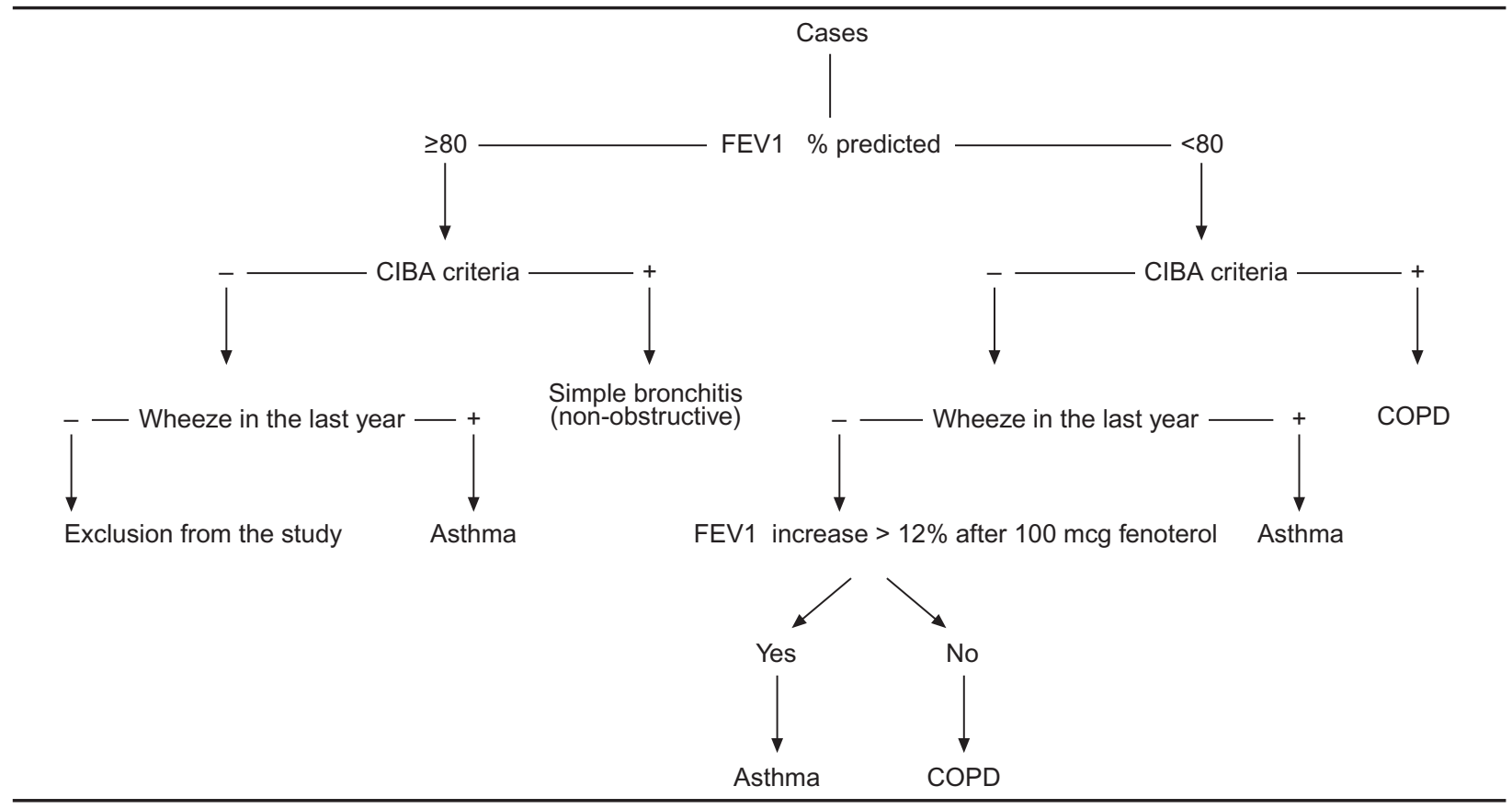

Figure 1 - Algorithm used for classifying respiratory patients into one of three diagnostic groups: asthma, COPD, non-obstructive bronchitis (simple bronchitis). " CIBA criteria: see Methods (Subjects).

tus by selected functional status indexes, with the intent of limiting the impact of subjectivity on the measured outcomes.

We aimed at verifying whether three expressions of chronic airway disease - COPD, asthma, chronic bronchitis uncomplicated by bronchial obstruction, i. e., simple bronchitis - differ in their effects on the health status of older patients. We hypothesized that asthma, due to its fluctuating course, would impact on the average health status less than COPD, and that simple bronchitis would have the least effect on health status because it does not affect airway caliber. With regard to individual dimensions of health status, we hypothesized that asthma mainly affected the quality of sleep because of its high rate of nocturnal exacerbation (15). Conversely, due to its respiratory and systemic effects, COPD was expected to outweigh asthma in limiting both basic and submaximal physical performance (16).

A further objective of the study was to compare the health status of respiratory patients with that of patients seeking medical care for non-respiratory diseases in an attempt to clarify the following topics: whether and to what extent a given respiratory condition has typical and/or predictable effects on health status; which functional status index can most effectively discriminate patients with different types of chronic airway disease; and what role comorbidity plays in conditioning the health status of older patients with airflow limitation.

\section{METHODS}

The Sa.R.A. (Salute Respiratoria nell'Anziano= Respiratory Health in the Elderly), a multi-center research project involving 24 geriatric or pulmonary institutions throughout Italy (17), was designed to assess various aspects of chronic airway disease in the elderly. The study protocol was approved by the Ethical Committees of the participating institutions. Patients gave their written informed consent to participate in the study.

The present analysis focuses on the effects of chronic airway disease on health status.

\section{Subjects}

Cases: Subjects were all outpatients aged 65 years and over, referred consecutively to the above-cited institutions between January 11996 and July 15 1997, because of a previous diagnosis of asthma or COPD or because of signs and/or symptoms compatible with either diagnosis. Diagnoses were checked by a diagnostic protocol based on a modified International Union Against Tubercolosis and Lung Diseases (IUATLD) questionnaire and on spirometry followed by the reversibility test $(17,18)$. Data collected were entered into an algorithm aimed at classifying patients into three categories: COPD, asthma and simple bronchitis (Fig. 1). The last definition applies to subjects with symptoms of chronic bronchitis according to ClBA criteria but FEV $1>80 \%$ of the predicted value. Note that the CIBA criteria define chronic bronchitis as "the con- 
dition of subjects with chronic or recurrent mucus secretion into the bronchial tree", i.e., a condition "occurring on most days of at least three months of the year for at least two successive years" (19).

Controls: We recruited subjects aged 65 years and over consecutively attending the outpatient geriatric departments in the same time period, but not reporting any previous diagnosis of respiratory disease or any symptoms or signs compatible with such a diagnosis.

Exclusion criteria from both study and control groups were: severe liver failure, corresponding to $\mathrm{B}$ or $\mathrm{C}$ grades of the Child index (20); New York Heart Association (NYHA) class III or IV cardiac failure (21); severe renal failure (serum creatinine $\geq 2 \mathrm{mg} / \mathrm{L}$ ); cognitive and/or sensory impairment severe enough to prevent multidimensional assessment; occurrence of a major psychosocial event (e.g., bereavement) within the past 6 months; hospitalization for any reason within the past 6 months.

\section{Procedures}

Subjects were investigated according to a protocol covering the following domains: social and environmental aspects; smoking history; pharmacological therapy, which was assessed and codified according to a previously validated method $(22,23)$; comorbid diseases, which were classified following the International Disease Classification, $9^{\text {th }}$ revision, Clinical Modification, and combined to compute Charlson's Index of comorbidity (24, 25); anthropometric data [(weight, height, Body Mass Index (BMI=weight/height $\left.\left.{ }^{2}\right)\right]$; waist-to-hip ratio, and occiput-wall distance (26-28). The last index reflects the degree of kyphosis of the upper thoracic and cervical spine, which is a correlate of both poor balance and osteoporotic vertebral fractures $(26,27)$.
Spirometry was performed by a fully-computerized water-sealed Stead-Wells spirometer (Baires, Biomedin, Padova, Italy) meeting the ATS 1994 recommendations for diagnostic spirometry (29). Patients having FEV1<80\% of the predicted value underwent a reversibility test with inhaled fenoterol, $100 \mathrm{mcg}$ (17).

The following health outcomes were measured:

1) The Barthel Index was used to explore independence in basic activities of daily living (30). The final score may range between 0 (complete dependence) and 100 (complete independence).

2) The 6-min walked distance was assumed to be a measure of submaximal physical performance (31). Patients were asked to walk back and forth at their maximal pace down a 20 -m long hospital corridor. They were allowed to rest if they felt exhausted, but the time spent resting could not be made up. Standard encouragement was given on each lap. The distance cumulatively covered in $6 \mathrm{~min}$ was recorded for analysis.

3) Mini-Mental State Examination (MMSE) was used to quantify cognitive performance. Normal subjects usually score 24 or more (32).

4) The 15-item Short Form of the Geriatric Depression Scale (15-GDS) was administered to assess affective status. A score over 5 is considered to be diagnostic of depressed mood (33).

5) The Established Populations for Epidemiological Studies of the Elderly (EPESE) questionnaire was used to rate sleep disturbances (34). The frequency of four types of sleep disturbances (difficulty initiating sleep, waking at night, feeling of tiredness after morning waking, waking too early) is graded according to the following 5-step score: $0=$ never; $1=$ occasionally (less than once a week);

Table 1 - Differences in sociodemographic, anthropometric and clinical variables among groups.

\begin{tabular}{|c|c|c|c|c|c|}
\hline & $\begin{array}{l}\text { Asthma } \\
\mathbf{N}=198\end{array}$ & $\begin{array}{l}\text { COPD } \\
\mathrm{N}=\mathbf{2 2 8}\end{array}$ & $\begin{array}{c}\text { Simple bronchitis } \\
\qquad \mathbf{N}=91\end{array}$ & $\begin{array}{l}\text { Controls } \\
\mathrm{N}=\mathbf{1 0 8 4}\end{array}$ & \\
\hline Age, M (SD) & $72.7(6)$ & $72.9(5.7)$ & $73.7(5.7)$ & $73.6(6.3)$ & $\mathrm{F}=1.55, p=0.2$ \\
\hline Sex, \% females & 53.5 & 16.9 & 29.3 & 48.8 & $\chi=117, p<0.001$ \\
\hline No heating, \% & 7 & 7.9 & 3 & 2.8 & $\chi=17.4, p<0.001$ \\
\hline Living alone, \% & 31.4 & 22.7 & 25.5 & 30.3 & $\chi=6.31, p=0.097$ \\
\hline Lower formal education, \% & 77.2 & 87.7 & 82.7 & 77.4 & $\chi=13.1, p=0.004$ \\
\hline Smokers, (no): -never & 91 & 27 & 26 & 516 & \\
\hline -actual & 21 & 42 & 23 & 101 & $\chi=144.5, p<0.001$ \\
\hline -previous & 84 & 161 & 50 & 367 & \\
\hline Pack-years, M (SD)* & $20.8(32.8)$ & $46.2(40.4)$ & $26.3(30.2)$ & $14(23.7)$ & $\mathrm{F}=8.03, p<0.001$ \\
\hline $\mathrm{BMI}, \mathrm{Kg} / \mathrm{cm}^{2}, \mathrm{M}(\mathrm{SD})$ & $27.9(4.9)$ & $25.6(4)$ & $26.2(4.3)$ & $26.4(4.2)$ & $\mathrm{F}=11.52, p<0.001$ \\
\hline $\mathrm{BMI}<22, \mathrm{Kg} / \mathrm{cm}^{2}, \%$ & 7.6 & 17.8 & 9 & 15.5 & $\chi=11.3, p=0.01$ \\
\hline Waist-to-hip ratio, M (SD) & $0.95(0.11)$ & $0.97(0.096)$ & $0.94(0.083)$ & $0.95(0.13)$ & $\mathrm{F}=3.45, p=0.02$ \\
\hline Index of comorbidity, M (SD) & $1.55(1.45)$ & $1.45(1.38)$ & $1.9(1.5)$ & $1.5(1.3)$ & $\mathrm{F}=2.86, p=0.04$ \\
\hline Drugs used, no., M (SD) & $3.6(2.1)$ & $3.9(2.2)$ & $2.9(1.9)$ & $2.1(1.7)$ & $\mathrm{F}=75.9, p<0.001$ \\
\hline
\end{tabular}

$\chi=$ chi square; * (no. of cigarettes smoked daily) / $20 \mathrm{x}$ (no. of years of smoking). 
Table 2 - Differences in health outcome variables (rows 2 to 6) and respiratory function indexes (rows 7 to 9) among groups.

\begin{tabular}{|c|c|c|c|c|c|}
\hline & $\begin{array}{l}\text { Asthma } \\
\mathbf{N}=198\end{array}$ & $\begin{array}{l}\text { COPD } \\
\mathbf{N}=\mathbf{2 2 8}\end{array}$ & $\begin{array}{c}\text { Simple bronchitis } \\
\mathrm{N}=91\end{array}$ & $\begin{array}{l}\text { Controls } \\
\mathrm{N}=1084\end{array}$ & $\mathbf{F}, \boldsymbol{p}$ \\
\hline Barthel Index, M (SD) & $92.5(8.5)$ & $92.2(7.3)$ & $95(5.1)$ & $94.7(6.7)$ & $11.9,<0.001$ \\
\hline 6' walked distance, $\mathrm{m}, \mathrm{M}$ (SD) & $304(127)$ & $300(123)$ & 346 (119) & $336(125)$ & $7.56,<0.001$ \\
\hline MMSE, M (SD) & $27.1(3.4)$ & $26.4(3.7)$ & $26.9(3.8)$ & $27(3.3)$ & $2.14,=0.09$ \\
\hline GDS, M (SD) & $4.3(3.6)$ & $4.2(3.1)$ & $4.7(3.9)$ & $3.6(3.3)$ & $6.12,<0.001$ \\
\hline Index of disturbed sleep, M (SD) & $4.9(4.6)$ & $3.9(4.5)$ & $4.4(4.3)$ & $3.6(4)$ & $5.66,<0.001$ \\
\hline FEV1/FVC, M (SD) & $62.5(13.8)$ & $40.5(14.2)$ & $71.2(9.1)$ & $75.5(8.6)$ & $369,<0.001$ \\
\hline FEV1, \% predicted, M (SD) & $70.1(24.4)$ & $51.6(16.3)$ & $98.8(14.8)$ & $99.8(19.6)$ & $435,<0.001$ \\
\hline FVC, \% predicted, M (SD) & $82.1(20.9)$ & $75.6(17.7)$ & $101.1(12.4)$ & $97.1(17.6)$ & $116,<0.001$ \\
\hline
\end{tabular}

$2=$ sometimes (once or twice a week); $3=$ often (three times or more in the week); $4=$ always. Thus, the global score can range between 0 and 16 (34).

No disease-specific instrument was used for the present analysis, because it could not be applied to non-respiratory patients.

\section{Statistical analysis}

The Epi Info (CDC, Atlanta, USA, and WHO, Geneva, Switzerland) and BMDP (University of California, Berkeley, California) statistical packages were used.

Differences among groups in the distribution of collected variables were assessed by the $\chi^{2}$ test for dichotomous data and by analysis of variance for continuous data.

Health outcome variables were entered into a discriminant analysis aimed at identifying subjects with similar health status profiles, as defined by the combination of scores achieved on individual indexes, within each group. The greater this fraction of patients in a given group, the more typical the health status profile of that group may be considered. The rate of concordance between actual group membership, as defined by the se- lecting criteria, and functional group membership, resulting from health status profiles, was computed. In a 4 by 4 cross-tabulation, corresponding to the four actual groups by the four functional groups, a rate of concordance of up to $25 \%$ was expected to occur by chance, whereas a figure greater than $25 \%$ for a given actual group was consistent with that group having a distinctive health status profile. The strength of the discriminant function was assessed by Wilk's lambda, which is a measure of significance of the discriminant function (35). The contribution of any individual variable to the discriminant function was assumed to be proportional to the absolute value of the coefficient of correlation between the discriminating variable and the discriminant function (35).

\section{RESULTS}

Twenty-five respiratory patients could not satisfactorily perform and 6 refused to perform spirometry, and were thus excluded from the study. The corresponding figures for control groups were 67 and 6 . Of the remaining 1501 patients, 198 qualified as being affected by asthma, 228 by COPD, 91 by simple bronchitis, and 1084 by

Table 3 - Results of discriminant analysis: canonical discriminant functions (upper panel) and determinants of discriminant functions (lower panel).

A. Canonical discriminant functions

\begin{tabular}{ccccc} 
Function & Eigen-value & \% of variance & Wilk's lambda & Significance \\
\hline 1 & 0.046 & 68.8 & 0.935 & $p<0.001$ \\
2 & 0.015 & 21.7 & 0.979 & $p<0.001$ \\
3 & 0.006 & 9.5 & 0.994 & $p<0.03$
\end{tabular}

B. Contribution of individual health outcomes to discriminant functions. Absolute value of standardized coefficients of correlation within functions reflects strength of correlation.

Function 1

\begin{tabular}{lccc}
\hline Barthel Index & 0.861 & 0.185 & 0.239 \\
6' walked distance & 0.249 & 0.364 & -0.645 \\
MMSE & -0.158 & 0.422 & 0.574 \\
GDS & -0.106 & 0.862 & -0.523 \\
Quality of sleep & -0.169 & 0.274 & 0.628 \\
\hline
\end{tabular}


Table 4 - Results of discriminant analysis: cross-tabulation of actual group membership, as defined by selection criteria (rows), and functional group membership, as defined by health outcome profile (columns).

\begin{tabular}{l|cccc}
\hline \multicolumn{5}{c}{ Functional group \% } \\
\hline Actual group, \% & Asthma & COPD & Simple bronchitis & Controls \\
\hline Asthma & 25.6 & 19.3 & 23.9 & 31.3 \\
COPD & 17.2 & 28.1 & 22.7 & 32 \\
Simple bronchitis & 11 & 8.8 & 42.9 & 37.4 \\
Controls & 14.3 & 10.1 & 25.1 & 50.4 \\
\hline
\end{tabular}

Box's $M=177.5, p<0.001$.

chronic non-respiratory diseases (controls). Control patients most commonly suffered from coronary artery disease-myocardial infarction (13.9\%), congestive heart failure $(6.3 \%)$, peripheral arterial vascular disease $(4.1 \%)$, osteoarthritis $(26.8 \%)$, diabetes mellitus $(12 \%)$, neurologic diseases (7.1\%) and malignant tumors (5.2\%).

Demographic, anthropometric, and clinical variables are listed in Table 1. Groups were comparable by age, whereas males, ex-smokers and current smokers prevailed in the COPD and simple bronchitis groups. These two groups were also characterized by lower formal education. Seven percent of asthmatics and $7.9 \%$ of COPD patients, but only $3 \%$ of simple bronchitis and $2.7 \%$ of control patients, lacked a domestic heating system $(p<0.001)$. COPD patients had the lowest average $\mathrm{BMI}$ and the highest fraction of subjects with $\mathrm{BMI}<22 \mathrm{Kg} / \mathrm{cm}^{2}(17.8 \%)$, but the highest waist-to-hip ratio, which is an index of central obesity. The simple bronchitis group was characterized by the highest index of comorbidity; no differences were found among the remaining groups.

Table 2 compares health outcome variables and respiratory function indexes of groups. The asthma and COPD groups had comparable impairment in physical function, as reflected by both the Barthel Index and 6-min walked distance; performances were poorer than those achieved by simple bronchitis and control subjects. All groups had similar MMSE scores. The highest and lowest average score on the 15-GDS characterized simple bron- chitis (4.7 \pm 3.9$)$ and control groups (3.6 \pm 3.3$)$, respectively. Asthmatics had the worst quality of sleep. Bronchial obstruction was mild to moderate in the majority of COPD patients: only $6 \%$ had a FEV $1<35 \%$ of that predicted.

The results of discriminant analysis are listed in Tables 3 and 4 . Three discriminant functions were obtained, the first and second of which explained 68.8 and $21.7 \%$ of the variance, respectively (Table 3, upper panel). The significant Wilk's lambda coefficients state that discriminant functions reflect real differences among groups and not sampling variability. The high Wilk's lambda values also demonstrate that a large proportion of total variance in the discriminant scores is explained by variance within individual groups. An index exploring physical fitness, the Barthel Index, and one reflecting mood, the 15-GDS, were the main determinants of discriminant functions 1 and 2 , respectively. The second most important determinant of function 1, the 6-min walked distance, further explored physical fitness (Table 3 , lower panel).

Table 4 cross-tabulates actual group membership, as defined by classificatory criteria, and functional group membership, resulting from the distribution of explored health outcomes. Only 28\% of COPD and $26 \%$ of asthma patients (actual groups) were classified as belonging to the COPD and asthma functional groups, respectively. This finding shows that asthma and COPD are not characterized by distinctive health status. The $43 \%$ and $50 \%$ degree of agreement between actual and functional group

Table 5 - Distribution of health outcomes in respiratory patients, subgrouped according to whether individual health outcome profiles conform to (C) or differ from (D) group-specific profiles.

\begin{tabular}{lccc|ccc|crc}
\hline & \multicolumn{3}{c}{ Asthma } & \multicolumn{3}{c|}{ COPD } & \multicolumn{3}{c}{ Simple bronchitis } \\
& C & D & $\boldsymbol{p}$ & C & D & $\boldsymbol{p}$ & C & D & $\boldsymbol{p}$ \\
\hline Barthel Index & $93.5(6.8)$ & $94.3(4.5)$ & 0.47 & $85(6.9)$ & $96(2.6)$ & $<0.001$ & $94.6(5.1)$ & $96.4(2.1)$ & 0.47 \\
6' walked distance, m & $248(109)$ & $325(128)$ & $<0.001$ & $265(112)$ & $325(119)$ & $<0.001$ & $385(127)$ & $328(104)$ & 0.05 \\
MMSE & $28.2(2.2)$ & $27.3(2.8)$ & 0.054 & $25.7(3.0)$ & $27.4(2.5)$ & $<0.001$ & $27.7(2.6)$ & $27.4(2.8)$ & 0.65 \\
GDS & $4.2(3.2)$ & $4.3(3.7)$ & 0.89 & $4.4(3.2)$ & $4.1(3.1)$ & 0.59 & $7.8(3.7)$ & $2.6(2.3)$ & $<0.001$ \\
Index of disturbed sleep & $8.4(4.0)$ & $3.6(4.0)$ & $<0.001$ & $3.4(4.4)$ & $4.1(4.4)$ & 0.29 & $6.3(4.9)$ & $2.9(3.1)$ & $<0.001$ \\
\hline
\end{tabular}

Data are expressed as means; standard deviation in brackets. 
membership for simple bronchitis and control subjects is much higher than the 25\% figure expected on the basis of chance classification. Both simple bronchitis and control subjects had low percentages of functional classifications in the asthma and COPD groups.

The distribution of health outcomes in respiratory patients subgrouped according to whether individual health outcome profiles conformed to $(C)$ or differed from $(D)$ group-specific profiles is listed in Table 5. A typical group-specific health outcome profile was characterized by lower 6-min walked distance and greater index of disturbed sleep in asthmatics, and by worse performance on the Barthel Index, the 6-min walking test and the MMSE in COPD patients. Depressed mood and disturbed sleep were the hallmarks of the group-specific health outcome profile of simple bronchitis patients.

Patients with and without group-specific health outcome profiles had comparable demographic, respiratory function and clinical variables in both asthma and simple bronchitis groups. A longer occiput-wall distance $(7.5 \pm 3.8$ us $6.1 \pm 3.7 \mathrm{~cm}, p<0.05)$, higher comorbidity index $(1.5+1.4$ vs $1.1+1.2, p<0.03)$ and more severe chronic obstruction (FEV1\% $=46.3+15.4$ vs $54.1+16.5, p<0.01$ ) were associated with group specificity, i.e., with worse health outcome profiles, in the COPD group.

\section{DISCUSSION}

This study shows that the selected dimensions of health status were differently affected by asthma and COPD in the elderly. However, neither asthma nor COPD were associated with a distinctive health status, as if their effects on the health status of older patients were frequently undistinguishable from those of simple bronchitis or non-respiratory diseases. The best health status characterized patients with simple bronchitis, i.e., with symptoms of bronchitis in the absence of airflow limitation, whereas the worst was observed in the COPD subset characterized by greater comorbidity and more severe airflow limitation, but not by older age.

The great heterogeneity in health status profiles within COPD and asthma groups probably reflects the complex interaction of factors conditioning disease-related health status in the elderly. Indeed, indexes of physical fitness, which explained most of the variance among groups, partially depended upon non-respiratory clinical variables: a longer occiput-wall distance, which reflects the severity of osteoporosis, and a higher index of comorbidity characterized COPD patients with worse health outcome profiles. Health status was unrelated to social or demographic variables, probably because the vast majority of COPD and asthma patients suffered from mild to moderate chronic airflow limitation, lived with their relatives, and could benefit by informal support. In more advanced stages of the respiratory disease, social factors are expected to impact more on the provision of help and, consequently, on health out- comes. Lastly, the relatively narrow age interval, due to the selection criteria, probably contributed toward weakening the relationship between age and health status. However, results from previous studies on older COPD populations support the conclusion that FEV1 limitation outweighs age as a negative correlate of health status $(12,36)$.

Depressed mood and disturbed sleep were the main components of health outcome profiles in simple bronchitis, but not in COPD. Thus, psychological problems seem to account for most of the variability in health status prior to the onset of airflow limitation; thereafter, physical limitation and cognitive impairment characterize patients with poorer health status.

The health outcome profiles of asthmatics differed from those of COPD patients because they strictly reflected quality of sleep and 6-min walked distance. The circadian rhythm of airflow limitation, leading to mainly nocturnal exacerbations of asthma, explains the former relationship (37). Most asthmatics were completely independent in activities of daily living, so that inter-individual differences could be seen to emerge in the 6-min walking test but not in the Barthel Index.

The differences in health status between simple bronchitis and COPD or asthma patients testify to the lack of a definite impact of the respiratory condition on health status prior to the onset of airflow limitation. When compared with simple bronchitis patients, both COPD and asthma patients had worse health status; however, this does not translate into a distinctive health status profile being highly prevalent within any of these groups. Both the choice of using a control non-respiratory population representative of usual and not of successful aging and the heterogeneous nature of COPD and asthma probably explain this seeming paradox (38-40).

This study has some limitations which deserve to be cited: 1) Distinction of asthma from COPD in the elderly is not easy $(41,42)$. Our algorithm represents a logical attempt at classifying these patients, but some weaknesses cannot be excluded. 2) The strength of a discriminant model depends largely upon the adequacy of the discriminating variables. We used assessment instruments which had been repeatedly validated in broad geriatric populations, but not in elderly respiratory patients, except for a study limited to 135 asthmatics (43). 3) The present results apply to patients with mild to moderate chronic airway disease, because severely diseased patients, whose hallmarks are greater comorbidity and lower physical capabilities, are underrepresented in this study (44).

\section{CONCLUSIONS}

Large inter-individual differences exist in disease-related health status among elderly patients with chronic respiratory diseases. Future research should aim at verifying whether differences in health status have prognostic implications as well as identifying the assessment instruments 
achieving the best discrimination. The recent demonstration that selected dimensions of quality of life are highly correlated with the frequency of COPD exacerbations emphasizes the need to pursue this research line (45). Eventually, close follow-up of these patients will allow us identify the meaning of clinical conditions, such as simple bronchitis or COPD with partially reversible obstruction, the evolution of which cannot be predicted on the basis of presently available knowledge.

\section{APPENDIX}

\section{The SaRA Study Group: list of participants}

Coordinators: V. Bellia (Palermo), F. Rengo (Napoli).

Scientific Committee: R. Antonelli Incalzi (Taranto), V. Grassi (Brescia), S. Maggi (Padova), G. Masotti (Firenze), G. Melillo (Napoli), D. Olivieri (Parma), M. Palleschi (Roma), R. Pistelli (Roma), M. Trabucchi (Roma), S. Zuccaro (Roma).

Participating centers, principal investigator and associated investigators (in brackets):

(1) Divisione Medicina I, Ospedale Geriatrico INRCA, Ancona: D.L. Consales (D. Lo Nardo, P. Paggi); (2) Divisione Geriatria, Ospedale Civile, Asti: F. Goria (P. Fea, G. Iraldi, R. Corradi); (3) Cattedra Geront. e Geriatria, Policlinico Universitario, Bari: A. Capurso (R. Flora, S. Torres, G. Venezia, M. Mesto); (4) V Divisione Geriatria, Ospedale Malpighi, Bologna: S. Semeraro (L. Bellotti, A. Tansella); (5) I Divisione Medicina Generale, Ospedale Civile, Brescia: V. Grassi (S. Cossi, G. Guerini, C. Fantoni, M. De Martinis, L. Pini); (6) Clinica Pneumologica, Fondazione "E. Maugeri", Telese (BN): G. Melillo (R. Battiloro, C. Gaudiosi, S. De Angelis); (7) Istituto Medicina Interna e Geriatria, Ospedale Cannizzaro, Catania: L. Motta (I. Alessandria, S. Savia); (8) Istituto Gerontologia e Geriatria, Ospedale Ponte Nuovo, Università di Firenze: G. Masotti (M. Chiarlone, S. Zacchei); (9) Divisione Geriatria, Ospedale Morgagni, Forli: V. Pedone (D. Angelini, D. Cilla); (10) Divisione Geriatria, Ospedale Galliera, Genova: E. Palummeri (M. Agretti, P. Costelli, D. Torriglia); (11) Gruppo Ricerca Geriatrica, Ospedale Richiedei, Gussago (BS): M. Trabucchi (P. Barbisoni, F. Guerini, P. Ranieri); (12) Divisione Geriatria, Ospedale Generale, L'Aquila: F. Caione (D. Caione, M. La Chiara); (13) I Divisione Geriatria, Ospedale San Gerardo, Monza: G. Galetti (A. Cantatore, D. Casarotti, G. Anni). (14) Cattedra Gerontologia e Geriatria, Università Federico II, Napoli: F. Rengo (F. Cacciatore, A.I. Pisacreta, C. Calabrese); (15) Istituto Medicina Interna, Ospedale Geriatrico, Padova: G. Enzi (P. Dalla Montà, S. Peruzza, P. Albanese, F. Tiozzo); (16) Istituto Malattie Apparato Respiratorio, Ospedale Rasori, Parma: D. Olivieri (V. Bocchino, A. Comel, N. Barbarito); (17) Istituto Gerontologia e Geriatria, Policlinico Monteluce, Perugia: U. Senin (F. Arnone, L. Camilli, S. Peretti); (18) Divisione Geriatria, Ospedale Israelitico, Roma: S.M. Zuccaro (M. Marchetti, L. Palleschi); (19) Divisione Geriatria, Ospedale Generale Addolorata, Roma: M. Palleschi (C. Cieri, F. Vetta); (20) Istituto Medicina Interna e Geriatria, Policlinico A Gemelli, Roma: P.U. Carbonin (F. Pagano, P. Ranieri, C. Romano); (21) Istituto Semeiotica Medica e Geriatria, Policlinico Le Scotte, Siena: S. Forconi (G. Abate, G. Marotta, E. Pagni); (22) Fondazione San Raffaele, Cittadella della Carità, Taranto: R. Antonelli-Incalzi (C. Imperiale, C. Spada); (23) Cattedra Gerontologia e Geriatria, Ospedale Maggiore, Milano: C. Vergani (G. Giardini, M.C. Sandrini, I. Dallera); (24) Cattedra Malattie Apparato Respiratorio, Ospedale V. Cervello, Palermo: V. Bellia (F. Catalano, N. Scichilone, S. Battaglia).

Coordinating Center: Istituto di Medicina Generale e Pneumologia, Cattedra Malattie Apparato Respiratorio, Università degli Studi di Palermo, Palermo, Italy.

\section{ACKNOWLEDGEMENTS}

The Sa.R.A. Project is generously supported by a research fund from Boehringer Ingelheim Italia, which is gratefully acknowledged.

\section{REFERENCES}

1. Testa MA, Simonson DC. Assessment of quality of life outcomes. N Engl J Med 1996; 334: 835-40.

2. Antonelli Incalzi R, Gemma A, Capparella O, et al. Predicting mortality and length of stay of geriatric patients in an acute care general hospital. J Gerontol 1992; 47: M35-9.

3. Gerardi DA, Lovett L, Benoit-Connors ML, Reardon JZ, ZuWallack RL. Variables related to increased mortality following out-patient pulmonary rehabilitation. Eur Respir J 1996; 9: 431-5.

4. Chang VT, Thaler HT, Polyak TA, Kornblith AB, Lepore JM, Portenoy RK. Quality of life and survival: the role of multidimensional symptom assessment. Cancer 1998; 83: 173-9.

5. Tosteson AN. Quality of life in the economic evaluation of osteoporosis prevention and treatment. Spine 1997; 22 (Suppl 24): 58S-62S.

6. Konstam V, Salem D, Pouleur H, et al. Baseline quality of life as a predictor of mortality and hospitalization in 5,025 patients with congestive heart failure. SOLVD Investigations. Studies of Left Ventricular Dysfunction Investigators. Am J Cardiol 1996; 78: 890-5.

7. Earlam S, Glover C, Firdy C, Burke D, Allen-Mersh TG. Relation between tumor size, quality of life, and survival in patients with colorectal liver metastasis. J Clin Oncol 1996; 14: 171-5.

8. Curtis R, Martin DP, Martin TR. Patient-assessed health outcomes in chronic lung disease. Am J Respir Crit Care Med 1997; 156: 1032-9.

9. Jones PW, Bosh TK. Quality of life changes in COPD patients treated with salmeterol. Am J Respir Crit Care Med 1997; 155: 1283-9.

10. Wijkstra PJ, Van Altena R, Kraan J, Otten V, Postma DS, Koeter GH. Quality of life in patients with chronic obstructive pulmonary disease: short-term effects on exercise endurance and health status. Eur Respir J 1994; 7: 269-73.

11. Albrecht GL, Devlieger PJ. The disability paradox: high quality of life against all odds. Soc Sci Med 1998; 48: 977-88.

12. Ketelaars CAJ, Schlosser MAG, Mostert R, Huyer Abu-Saad H, Halfens RJG, Wouters EFM. Determinants of health-related quality of life in patients with chronic obstructive pulmonary disease. Thorax 1996; 51: 39-43.

13. Ferrer M, Alonso J, Morera J, et al, for the Quality of Life of Chronic Obstructive Pulmonary Disease Study Group. Chronic obstructive pulmonary disease stage and health-related quality of life. Ann Intern Med 1997; 127: 1072-9.

14. Dyer CAE, Stockley RA, Sinclair AJ. Quality of life in elderly subjects with a diagnostic label of asthma from general practice registers. Eur Respir J 1999; 14: 39-45.

15. Bellia V, Pistelli R, Filippazzo G, et al. Prevalence of nocturnal asthma in a general population sample: determinants and effect of aging. J Asthma 2000; 37: 595-602.

16. Debigare R, Cote Ch, Maltais F. Peripheral muscle wasting in chronic obstructive pulmonary disease: clinical relevance and mechanisms. Am J Respir Crit Care Med 2001; 164: 1712-7.

17. Bellia V, Pistelli R, Catalano F, et al, and the Sa.R.A Study group. Quality control of spirometry in the elderly: the Sa.RA. Study. Am J Respir Crit Care Med 2000; 161: 1094-100.

18. Abramson MJ, Hensley MJ, Saunders NA, Wlodarczyk JJ. Evaluation of a new asthma questionnaire. J Asthma 1991; 28: 129-39.

19. Ciba Guest Symposium report. Terminology, definitions and 
classification of chronic pulmonary emphysema and related conditions. Thorax 1959; 14: 286-99.

20. Child CG. The liver and portal hypertension. Philadelphia: WB Saunders, 1964: 50.

21. Criteria Committee, New York Heart Association, Inc. Diseases of the heart and blood vessels. Nomenclature and criteria for diagnosis, 6th ed. Boston: Little Brown and Co., 1964: 114.

22. Carbonin PU, Pahor M, Bernabei R, Sgadari A. Is age an independent risk factor of adverse drug reactions in hospitalized medical patients? J Am Geriatr Soc 1991; 46: 1305-14.

23. World Health Organization. International Drug Monitoring: The role of the hospital. WHO Tech Rep No. 452, 1969.

24. International Classification of Disease, 9th revision, Clinical Modification. Washington: Public Health Service, Health Care Finance Administration, 1980.

25. Charlson ME, Pompei P, Ales KL, Mackenzie CR. A new method of classifying prognostic comorbidity in longitudinal studies: development and validation. J Chronic Dis 1987; 40: 373-83.

26. O'Brien K, Culham E, Pickles B. Balance and skeletal alignment in a group of elderly female fallers and nonfallers. J Gerontol 1997; 52: B221-6.

27. Lyles KW, Gold DT, Shipp KM, Pieper CF, Martinez S, Mulhausen PL. Association of osteoporotic vertebral fractures with impaired functional status. Am J Med 1993; 94: 596-601.

28. Rosmond R, Dallman MF, Bjorntorp P. Stress related cortisol secretion in men: relationship with abdominal obesity and endocrine, metabolic and hemodynamic abnormalities. J Clin Endocrinol Metab 1998; 83: 1842-5.

29. American Thoracic Society. Standardization of spirometry. 1994 Update. Am J Respir Crit Care Med 1995; 152: 1107-36.

30. Mahoney F, Barthel D. Functional evaluation: the Barthel Index. MD State Med J 1965; 14: 61-5.

31. Guyatt GH, Sullivan MJ, Thompson PJ, et al. The six minute walk: a new measure of exercise capacity in patients with chronic heart failure. Can Med Assoc J 1985; 132: 919-23.

32. Folstein M, Folstein S, McHugh P. Mini mental state: a practical method for grading the cognitive state of patients for the clinician. J Psychiatr Res 1975; 12: 189-98.
33. Yesavage J, Brink TL, Rose TL, et al. Development and validation of a geriatric depression screening scale: a preliminary report. J Psychiatr Res 1982; 17: 37-49.

34. Maggi S, Langlois JA, Minicuci N, et al. Sleep complaints in community-dwelling older persons: prevalence, associated factors, and reported causes. J Am Geriatr Soc 1998; 46: 161-8.

35. Brown GW. Discriminant analysis. Am J Dis Child 1984; 138: 395-400.

36. Renwick DS, Connolly MJ. Impact of obstructive airway disease on quality of life in older adults. Thorax 1996; 51: 520-5.

37. Turner-Warwick M. Epidemiology of nocturnal asthma. Am J Med 1988; 85 (Suppl 1B): 6-9.

38. National Center for Health Statistics, et al. Health Statistics on Older Persons, United States 1986. Vital and Health Statistics, Series 3, No. 25. DHHS Publication (PHS) 87-1409, Public Health Service. Washington, DC: Government Printing Office, 1987.

39. Petty TL, Weinman GG. Building a national strategy for the prevention and management of and research in chronic obstructive pulmonary disease. JAMA 1997; 277: 246-53.

40. Braman SS, Kaemmerlen JT, Davis SM. Asthma in the elderly. A comparison between patients with recently acquired and longstanding disease. Am Rev Respir Dis 1991; 143: 336-40.

41. Burr ML, Charles TJ, Seaton A. Asthma in the elderly: an epidemiological survey. BMJ 1979; 1: 1041-4.

42. Burrows B, Barbee RA, Cline MG, Knudson RJ, Lebowitz MD. Characteristics of asthma among elderly adults in a sample of the general population. Chest 1991; 100: 935-42.

43. Nejjari C, Tessier JF, Barberger-Gateau P, Jacqmin H, Dartiques JF, Salamon R. Functional status of elderly people treated for asthma-related symptoms: a population based case-control study. Eur Respir J 1994; 7: 1087-93.

44. Antonelli Incalzi R, Pedone C, Onder M, Pahor M, Carbonin PU, for the GIFA. Predicting length of stay of older patients with exacerbated chronic obstructive pulmonary disease. Aging Clin Exp Res 2001; 13: 49-57.

45. Seemungal TAR, Donaldson GC, Paul EA, Bestall JC, Jeffries DJ, Wedzicha JA. Effect of exacerbation on quality of life in patients with chronic obstructive pulmonary disease. Am J Respir Crit Care Med 1998; 157: 1418-22. 